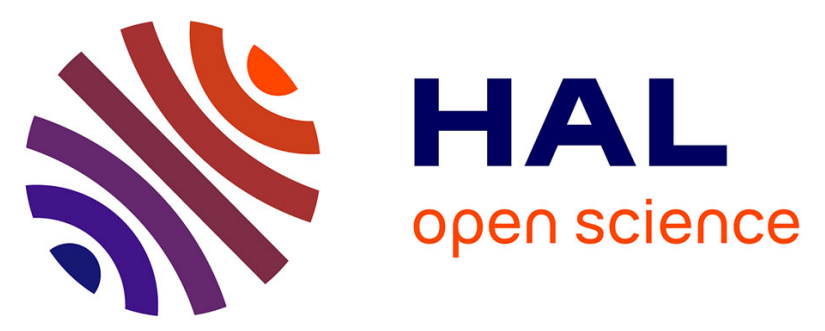

\title{
Alterations in ileal and colonic permeability by chronic intake of high-lipid diets enriched with omega-3, omega-6 or saturated fat
}

Jean Paul Lalles, Cécile Perrier, David Val-Laillet, Charles-Henri Malbert

\section{To cite this version:}

Jean Paul Lalles, Cécile Perrier, David Val-Laillet, Charles-Henri Malbert. Alterations in ileal and colonic permeability by chronic intake of high-lipid diets enriched with omega-3, omega-6 or saturated fat. Digestive disease week, May 2013, Orlando, United States. Gastroenterology, 144 (5) (Suppl.1), 2013, Gastroenterology. 10.1016/S0016-5085(13)63111-X . hal-01595089

\section{HAL Id: hal-01595089 \\ https://hal.science/hal-01595089}

Submitted on 3 Jun 2020

HAL is a multi-disciplinary open access archive for the deposit and dissemination of scientific research documents, whether they are published or not. The documents may come from teaching and research institutions in France or abroad, or from public or private research centers.
L'archive ouverte pluridisciplinaire HAL, est destinée au dépôt et à la diffusion de documents scientifiques de niveau recherche, publiés ou non, émanant des établissements d'enseignement et de recherche français ou étrangers, des laboratoires publics ou privés.

\section{다(1)(2)}

Distributed under a Creative Commons Attribution - ShareAlikel 4.0 International 
despite higher levels of apoptosis ( $\mathrm{p}=0.02$ ). Levels of $\mathrm{s} C \mathrm{CD} 14$ and IL- 6 were elevated during HIV infection. Soluble CD14 was elevated in both viremic $(\mathrm{p}=0.05)$ and ART-suppressed subjects ( $\mathrm{p}=0.0006$ ), and was positively correlated with levels of epithelial apoptosis (rho: $0.55, \mathrm{p}=0.04$ ) in suppressed subjects. Conclusions: HIV increases the levels of CEC apoptosis, which fail to normalize during suppressive ART in immunologic non-responders. The level of intestinal apoptosis was associated with innate immune activation. While the causal pathways remain unclear, strategies to improve persistently elevated levels of apoptosis and mucosal injury may ameliorate systemic inflammation during chronic HIV infection.

\section{Tu1746}

Activation of Snail via Reactive Oxygen Species Mediates AcetaldehydeInduced Disruption of Tight Junctions in CACO-2 Cell Monolayer Elhaseen Elamin, Ad Masclee, Freddy Troost, Jan Dekker, Daisy Jonkers

Background: Exposure to acetaldehyde is associated with intestinal barrier dysfunction and risk of malignant transformation in the gastro-intestinal tract. Epithelial-mesenchymal transition (EMT) is a developmental program shown to play a role in loss of epithelial integrity, cancer progression and metastases. EMT is induced by its transcription factor Snail, which downregulates E-cadherin expression. Recently, activation of EMT Snail has been found to mediate ethanol-induced intestinal barrier disruption. As acetaldehyde has an even higher potency to induce barrier dysfunction and is mutagenic, we hypothesized that acetaldehyde disrupts intestinal epithelial integrity by inducing oxidant-dependent Snail activation. Aims: To investigate the role of oxidative stress and Snail activation in acetaldehyde induced barrier disruption in an in vitro model of intestinal permeability. Methods: Caco2 monolayers were exposed from the luminal side to $25 \mu \mathrm{M}$ acetaldehyde $+/-100 \mu \mathrm{M} \mathrm{L}$ cysteine to inhibit ROS generation. Intestinal epithelial permeability, localization and expression of ZO-1, occludin, E-cadherin, $\beta$-Catenin were examined using TEER and FITC-D4 flux, immunofluorescence and ELISA, respectively. ROS generation and Snail activation were assessed by ELISA and immunofluorescence. Involvement of Snail was further addressed by inhibiting Snail using small interfering RNA (siRNA). Results: Exposure to acetaldehyde $25 \mu \mathrm{M}$ significantly increased the paracellular permeability (60\% decrease in TEER and 34\% increase in FITC-D4 flux vs. medium only-treated controls, both P $<0.0001$ ) in association with redistribution and decrease of tight junction (TJ) and adherens junction (AJ) protein levels. Acetaldehyde increased ROS generation by $40 \%$ and Snail phosphorylation by $30 \%$ (both $\mathrm{P}<0.0001$ vs. medium only-treated controls). These effects were attenuated by LCysteine ( $(\mathrm{P}<0.0001$ vs. acetaldehyde only-treated monolayers). Knock down of Snail by siRNA significantly attenuated acetaldehyde-induced changes and decrease in TJ and AJ proteins, improved TEER and decreased FITC-D4 permeation (all P $<0.05$ vs. non-specific Snail-transfected cells). Conclusions: Our data demonstrate that oxidative stress-mediated Snail phosphorylation is likely a novel mechanism contributing to the deleterious effects of acetaldehyde on intestinal barrier function. Identification of mechanisms involved in acetaldehyde-induced intestinal barrier dysfunction may provide new therapeutic targets for prevention of alcohol-related gut leakiness with subsequent development of liver disease and colon carcinogenesis.

\section{Tul747}

MRNAs for Ucpl and PRDM16 Are Detectable in Visceral Adipose of Obese Subjects Indicating Therapeutic Trans-Differentiation Potential

Aybike Birerdinc, Rohini Mehta, Katherine Doyle, Lei Wang, Tugba Keskin, Zahra

Younoszai, Ancha Baranova, Hazem A. Elariny, Amir H. Moazzez, Zobair M. Younossi

Background and Aim: Several lines of research have suggested brown adipose tissue (BAT) may be involved in obesity. Our aim was to profile the expression of BAT-specific UCPI and PRDM1 6 genes within the visceral adipose tissue depots in a cohort of obese individuals. Additionally, we profiled the levels of KCNRG which encodes a potassium current suppressor that may play a role in diet induced obesity. Methods: Obese patients undergoing bariatric surgery with clinical data, serum and adipose tissue samples were included. Visceral adipose tissue was collected at the time of bariatric surgery and snap frozen in liquid nitrogen. RNA was extracted from frozen adipose samples using the AURUM Total RNA Fatty and Fibrous Tissue Kits (Biorad) and cDNA was synthesized from $1 \mu \mathrm{g}$ of the total RNA using RT2 first strand kit (Qiagen). RT-PCR profiling was performed using validated primers for KCNRGvl (amplifies isoforms A and B), KCNRGv2 (specifically amplifies only isoform A), UCP1 and PRDM16. ACTB was used as the reference gene for data normalization. Mann-Whitney U test and chi-square test were performed on continuous and categorical data respectively. Spearman's rank-sum correlation coefficients and corresponding p-values were calculated. Results: A total of 14 subjects (35.7\% type 2 diabetes, BMI $46.7+/-12$, AST $24.9+/-8.9$ U/ L, ALT $34.4+/-15.7 \mathrm{U} / \mathrm{L}$ ) were included. Our analysis shows a significant positive correlation between the levels of UCPl gene expression and BMI $(r=0.367, p<0.019)$ as well as UCPl and the expression level of KCNRG isoform A $(r=0.636, p<0.026)$. Conclusion: The data from this pilot study suggest that mRNAs for UCP1 and PRDM16 are detectable in visceral adipose of obese subjects. Therefore, we believe that, at least some cells embedded in VAT, have a potential for trans-differentiation into brown adipocytes. Further studies in larger cohorts are warranted to better elucidate this possibility

\section{Tu1748}

Alterations in Ileal and Colonic Permeability by Chronic Intake of High-Lipid Diets Enriched With Omega-3, Omega- 6 or Saturated Fat Jean-Paul Lalles, Cecile Perrier, David Val-Laillet, Charles-Henri Malbert

Gut permeability is a key function often incriminated in the development of local and systemic inflammation. Chronic consumption of high-fat diets leads to obesity and inflammation. Data in mice revealed a causal increase in intestinal permeability to bacterial lipopolysaccharide (LPS) that may be responsible for adipose tissue development and inflammation (Cani et al, 2009). Whether intestinal or colonic barrier can be modulated by omega-6 (O6) or omega-3 (O3) PUFA in the context of high-fat diet-induced obesity is poorly understood.
Intestinal epithelial barrier in vitro is strengthened by both $\mathrm{O} 6$ and $\mathrm{O} 3$ fatty acids. In vivo, rats chronically fed high fat diets enriched in PUFA did not show different paracellular permeabilities in the jejunum, compared to rats fed a diet rich in saturated fat. Whether colonic permeability is altered has not been reported. We hypothesized that PUFA-enriched diets modulate gut permeability differentially according to permeability pathway and gut site. This hypothesis was tested in 16 obese adult minipigs fed for 2 months with iso-protein and iso-calorie high-fat (12\%) diets based on lard (saturated fat, S), sunflower oil (rich in O6), or fish oil (rich in O3). Ileal and colonic mucosa were collected and both para- and trans-cellular permeability were investigated with fluorescein isothiocyanate-dextran 4 and horseradish peroxidase in Ussing chambers. Gut permeability was assessed without and with an inflammatory challenge (E. coli LPS added at 0.1 and $10 \mu \mathrm{g} / \mathrm{ml}$ ). After two months of regimen, body weight was not different between diet groups. Ileal mucosa density was not affected by the diet. Ileal permeability to FD4 was not influenced by the diet in the basal state but it tended to be higher with O3 compared to O6 diet after LPS challenge (e.g. with LPS $0.1 \mu \mathrm{g} / \mathrm{ml}: 1272 \pm 389,775 \pm 355$ and $1723 \pm 389) \mathrm{ng} / \mathrm{cm} 2 / \mathrm{h} ; \mathrm{P}=0.095)$ Interestingly, basal colonic permeability to FD4 was higher with O3 compared to O6 diet $(692 \pm 165,509 \pm 150$ and $1032 \pm 165 \mathrm{ng} / \mathrm{cm} 2 / \mathrm{h}$ for diets based on saturated, O6 and $\mathrm{O} 3$ $\mathrm{FA}$, respectively; $\mathrm{P}<0.05)$. Dietary treatments did not affect basal ileal or colonic permeability to HRP. However, both ileal and colonic permeability to HRP tended to be higher with $\mathrm{O} 3$ than $\mathrm{O} 6$ diet after LPS challenge. Our data indicate that gut permeability is modulated by dietary FA source regionally and according to permeability pathway. Para-cellular permeability in the colon (but not in the ileum) was affected in the basal state by diet FA composition. Surprisingly, it was higher with the $\mathrm{O} 3$ as compared to O6-enriched diet. The same trends for differences between $\mathrm{O} 3$ and $\mathrm{O} 6$ diets on para- or trans-cellular permeability were also revealed under inflammatory condition.

\section{Tu1749}

Intestinal Sweet Taste Receptor and Transporter Expression, Post-Prandial Glycemia and Glucose Absorption in Morbidly Obese Subjects Nam Q. Nguyen, Tamara L. Debreceni, Bridgette Chia, Carly M. Burgstad, Melissa Neo, Adam M. Deane, Gary A. Wittert, Michael Horowitz, Richard L. Young

Intestinal sweet taste receptors (STR) sense luminal glucose and rapidly increase levels and function of the glucose transporters SGLT 1 and GLUT-2 in health. Increased expression of SGLT-1 and glucose absorption has been reported in obese rats; however, limited data exists on the expression of intestinal STR and glucose transporters in obese subjects. AIM: To determine the relationship between expression of intestinal STR and glucose transporters with postprandial glycemia and glucose absorption in morbidly obese subjects. METHODS: After an overnight fast, 16 morbidly obese subjects (4M:12F; $44 \pm 3 y r s$, BMI: $47 \pm 4 \mathrm{~kg} / \mathrm{m}^{2}$ ) and 11 healthy volunteers (11M:1F; $44 \pm 6 y r s, B M I: 25 \pm 1 \mathrm{~kg} / \mathrm{m}^{2}$ ) underwent an upper gastrointestinal endoscopy to obtain mucosal biopsies from the duodenum at baseline and $30 \mathrm{~min}$ after a duodenal glucose infusion (30g glucose with 3g 3-O-methyl-D-glucopyranose (3OMG) in $150 \mathrm{ml}$ water, infused at $5 \mathrm{ml} / \mathrm{min}$ ). Blood glucose and 3-OMG concentrations were assessed at baseline and over $240 \mathrm{~min}$ after duodenal glucose infusion. Levels of STR (T1R2) and glucose transporter (SGLT-1 and GLUT2) transcript were quantified in biopsies by absolute RT-PCR. Integrated glucose absorption was assessed by plasma 3-OMG area under the curve over 240 mins (AUC $240 \mathrm{n}$ ). RESULTS. Expression of intestinal TIR2 in morbidly obese subjects was $60 \%$ lower than that of healthy subjects at baseline $(\mathrm{P}=0.03)$ and remained lower 30 min after glucose infusion $(64 \%, P=0.02)$, however, expression of glucose transporters was similar between groups. Glucose infusion reduced intestinal expression of SGLT-1 by $26 \%(\mathrm{P}=0.01)$ in the morbidly obese but not in healthy subjects. In contrast, intestinal expression of TIR2 and GLUT2 was unchanged by acute glucose infusion in either group. Post-prandial blood glucose $(\mathrm{P}<0.0001)$ and plasma 3 -OMG concentrations $(\mathrm{P}=0.005)$ were both significantly higher in the morbidly obese than in healthy subjects. Intestinal expression of GLUT2 correlated negatively with BMI at baseline in the morbidly obese ( $\mathrm{r}=-0.65, \mathrm{P}=$ 0.006 ), while GLUT2 expression following glucose infusion correlated positively with glucose absorption (3-OMG AUC C 240 in $_{2} ;=0.68, \mathrm{P}<0.001$ ). Such relationships were not observed in healthy subjects. CONCLUSION: Despite reduced expression of intestinal STR at baseline and SGLT-1 following glucose in the morbidly obese, intestinal glucose absorption is increased with higher post-prandial glycemia. These findings suggest a potential dys-regulation of intestinal STR and/or glucose transporter function in the morbidly obese that promotes glucose absorption and therefore, provides a possible underlying mechanism of weight gain in obesity.

\section{Tu1750}

Inflammation and Oxidative Stress in the Small Intestine Are Associated With Insulin Resistance and Alterations in Lipid Homeostasis in Obese Subjects Alain Veilleux, Émilie Grenier, Picard Marceau, André C. Carpentier, Denis Richard, Emile Levy

Intestinal chronic inflammation and oxidative stress are commonly associated with inflammatory bowel diseases. However, much less is known about their contribution to additional disorders such as metabolic syndrome and diabetic dyslipidemia. This is of interest since chronic low-grade inflammation and oxidative stress have been previously shown to contribute to the development of insulin resistance and several metabolic alterations in the liver muscle and adipose tissue. Objective: To reveal the presence of inflammation and oxidative stress in the small intestine and their link to insulin resistance as well as to abnormalities in lipid homeostasis in obese subjects. Methods: Duodenal specimens obtained from 20 obese subjects undergoing bariatric surgery were paired for age, gender and BMI with or without insulin resistance, as defined by the homeostasis model assessment of insulin resistance (HOMA-IR) index. Inflammation and oxidative stress markers as well as synthesis and transport of lipids and apolipoproteins (apo) were measured in these samples. Results: Compared to insulin-sensitive (IS) subjects (HOMA-IR <3.2), insulin-resistant (IR) subjects (HOMA-IR > 7) were characterized by reduced intestinal insulin signaling (lower Akt Ser473 and AMPK Thr172 phosphorylation). They also displayed in their duodenum: (a) enhanced p38 MAPK Thr180/Tyr182 phosphorylation, involved in inflammatory cytokine signaling; (b) elevated protein expression ratio of the transcription factor NF- $\kappa B$ to its inhibitor $I \kappa B$, 